\title{
Impact of ultrasonic dispersion on the photocatalytic activity of titania aggregates
}

\author{
Hoai Nga Le ${ }^{* 1,2}$, Frank Babick ${ }^{3}$, Klaus Kühn ${ }^{1}$, Minh Tan Nguyen ${ }^{2}$, Michael Stintz ${ }^{3}$ \\ and Gianaurelio Cuniberti ${ }^{1,4,5}$
}

\author{
Full Research Paper \\ Address: \\ ${ }^{1}$ Institute for Materials Science and Max Bergmann Center of \\ Biomaterials, TU Dresden, 01062 Dresden, Germany, ${ }^{2}$ School of \\ Chemical Engineering, Hanoi University of Science and Technology, \\ 10000 Hanoi, Vietnam, ${ }^{3}$ Institute of Process Engineering and \\ Environmental Technology, TU Dresden, 01069 Dresden, Germany, \\ ${ }^{4}$ Dresden Center for Computational Materials Science (DCCMS), TU \\ Dresden, 01062 Dresden, Germany and ${ }^{5}$ Center for Advancing \\ Electronics Dresden, TU Dresden, 01062 Dresden, Germany \\ Email: \\ Hoai Nga Le ${ }^{*}$ - hnle@nano.tu-dresden.de \\ * Corresponding author \\ Keywords: \\ AOPs; reaction rate constant; turbidity; ultrasonic energy; wastewater \\ treatment \\ Beilstein J. Nanotechnol. 2015, 6, 2423-2430. \\ doi:10.3762/bjnano.6.250 \\ Received: 05 October 2015 \\ Accepted: 04 December 2015 \\ Published: 17 December 2015 \\ Associate Editor: N. Motta \\ (c) 2015 Le et al; licensee Beilstein-Institut. \\ License and terms: see end of document.
}

\begin{abstract}
The effectiveness of photocatalytic materials increases with the specific surface area, thus nanoscale photocatalyst particles are preferred. However, such nanomaterials are frequently found in an aggregated state, which may reduce the photocatalytic activity due to internal obscuration and the extended diffusion path of the molecules to be treated. This paper investigates the effect of aggregate size on the photocatalytic activity of pyrogenic titania (Aeroxide ${ }^{\circledR} \mathrm{P} 25$, Evonik), which is widely used in fundamental photocatalysis research. Well-defined and reproducible aggregate sizes were achieved by ultrasonic dispersion. The photocatalytic activity was examined by the color removal of methylene blue (MB) with a laboratory-scale setup based on a plug flow reactor (PFR) and planar UV illumination. The process parameters such as flow regime, optical path length and UV intensity are welldefined and can be varied. Our results firstly show that a complete dispersion of the P25 aggregates is not practical. Secondly, the photocatalytic activity is not further increased beyond a certain degree of dispersion, which probably corresponds to a critical size for which UV irradiation can penetrate the aggregate without significant obscuration.
\end{abstract}

\section{Introduction}

Advanced oxidation processes (AOPs) form a group of modern chemical technologies that rely on the generation of radical species and are considered to have high prospects for the oxi- dation, discoloration, mineralization, and degradation of organic pollutants [1,2]. Photocatalysis is an example of an AOP that has been effectively applied for the treatment of highly polluted 
water such as dye sewage $[3,4]$. Among the materials for this application, titanium dioxide $\left(\mathrm{TiO}_{2}\right)$ is a very promising photocatalyst because of its commercial availability, chemical and biological inertness, and because it has no known adverse health effects on humans $[5,6]$. Due to its large active surface area, the suspended $\mathrm{TiO}_{2}$ powder is favored [6].

Most slurry photocatalysts have been implemented in illuminated batch reactors [6-8] and follow Langmuir-Hinshelwood kinetics $[9,10]$. This research has focused on the materials aspects such as the structural properties (e.g., surface area, particle size, crystal composition, porosity) $[8,11]$ of pristine or modified photocatalysts $[2,5,12]$. However, many of these laboratory-scaled apparatus are inappropriate to be applied in well-defined conditions, making the application with available pilot photoreactors challenging. Besides, such particles often form aggregates $[5,13]$ whose properties differ from those of the primary particles, leading to misconceptions about the photocatalytic characteristics. In contrast to the photochemical aspect, in which aggregation has been more thoroughly discussed $[14,15]$, the effects of the aggregates/secondary particles on the photocatalytic applications are still inexplicit. While the higher photocatalytic activity of fine, primary particles (as a result of the larger surface area) has been investigated [4,16-18], the behavior and properties of the aggregates is not well understood.

This paper shows an engineering approach to study the aggregation in photocatalysts. The first part presents the experimental setup, which defines the process parameters. In addition, ultrasonic dispersion was used to disintegrate the P25 nano-photocatalyst as well as vary the size. The photocatalytic activity was examined by the discoloration of MB under UV irradiation.

\section{Experimental Materials}

All experiments were conducted with commercial titanium(IV) oxide powder (Aeroxide ${ }^{\circledR}$ P25, Evonik, CAS-No. 13463-67-7), which consists of an approximately 80/20 w/w rutile/anatase mixture.

MB (Merck, KGaA), a model substance in dye wastewater research [4,7], was chosen as the organic compound in the photocatalysis. The discoloration of MB, in consequence, indicates the photocatalytic properties of P25 [19].

\section{Experimental setup}

An industrial, photocatalytic implementation requires a photoreactor, which not only satisfies basic techniques of chemical engineering [10] but also ensures effective photon collecting [20].
An idea for a simple experimental setup was developed (Figure 1) with the following key points: (1) the artificial illuminator is planar and produces steady-state UV radiation of intensity similar to that of solar illumination $\left(20-30 \mathrm{~W} / \mathrm{m}^{2}\right)$ $[4,5,21]$; (2) the reactor is a rectangular cell, so that the influence of the UV intensity is only two-dimensional; (3) the thickness of the reactor is small to diminish the shielding effect in the non-illuminated region [22]; and (4) most importantly, the reactor operates based on a PFR as the absorbent tube in the solar collecting reactors [4,23-26].

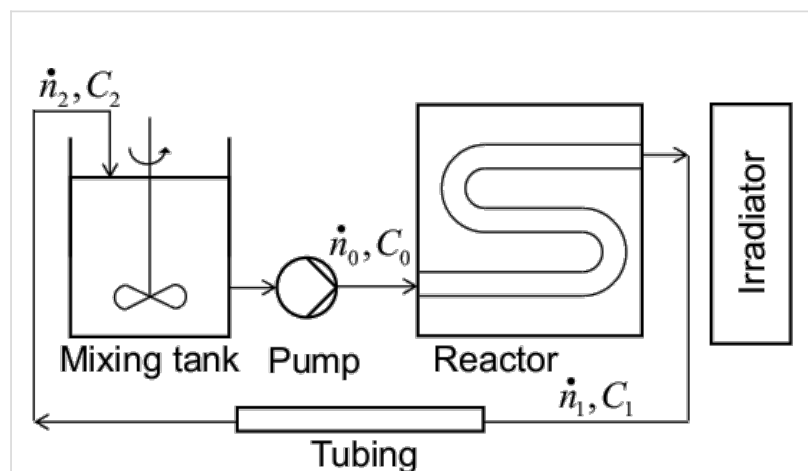

Figure 1: Schematic of the experimental setup consisting of a reservoir, pump, photoreactor and irradiator.

The setup design was then developed as shown in Figure 2. The reactor has a footprint size of $260 \times 180 \times 35 \mathrm{~mm}$ and includes six continuous channels, of which the size is $25 \times 120 \times 22 \mathrm{~mm}$ corresponding to a volume of $411 \mathrm{~mL}$. The illuminated surface of the reactor is $0.45 \mathrm{~cm}^{2}$ and is made from $3.3 \mathrm{~mm}$ Schott Borofloat $^{\circledR} 33$ glass, which has a UVA transmittance $>90 \%$, as specified by the manufacturer. The illuminating device was constructed by UMEX GmbH with six Phillips $8 \mathrm{~W}$ mercury fluorescent tubes with a mode wavelength value of $365 \mathrm{~nm}$. The UV intensity at the window of the reactor measured by an intensity meter (PCE-UV34) was 12.0-22.1 W/m² (Figure 2). The circulation of fluid is driven by a Micropump ${ }^{\circledR} 132-665-316$ pump allowing a flow rate of $2.82 \mathrm{~L} / \mathrm{min}$, corresponding to a Reynolds number in the channels of $\approx 1150$.

The appropriate process parameters such as flow regime, optical path length and average UV intensity are defined and can be adjusted. This setup allows the establishment of other specific constants, such as intensity- or flow-regime-based reaction rate constants for new investigations.

\section{Experimental determination of reaction rate constant}

Langmuir-Hinshelwood kinetics have been commonly applied to quantify the photocatalytic conversion of organic com- 


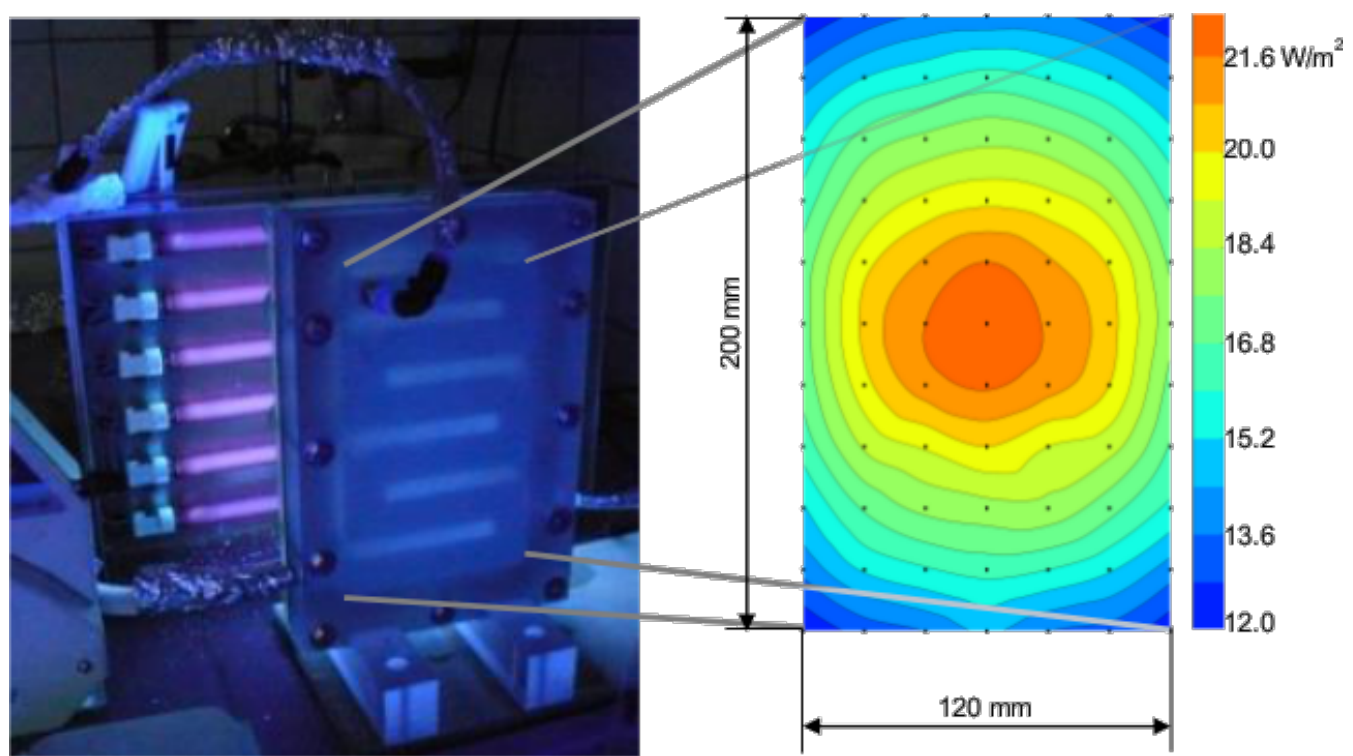

Figure 2: The photoreactor placed in front of the irradiator and the UV intensity distribution on the illuminated surface.

pounds in batch reactors $[9,10]$. For a new design based on a PFR, determining the reaction rate constant is required.

Since the change of the amount of organic compound $\mathrm{A}$ in the PFR is produced only by the reaction, the material balance is derived as [27]:

$$
\frac{d \dot{n}_{1}}{d V_{\mathrm{R}}}=\vartheta r
$$

where $n$ and $\vartheta$ are moles and the stoichiometric coefficient of species A, respectively, $V_{\mathrm{R}}$ is the volume of the PFR, $r$ is the reaction rate equal to $r=k C_{1}$, and $k$ is the reaction rate constant.

In the mixing tank, by assuming that the change of amount is due to the in- and outflows, and not due to the reaction, the material balance follows [27]

$$
\frac{d n_{0}}{d t}=-\dot{n}_{0}+\dot{n}_{2}
$$

The concentration of species A is specified as

$$
C_{0}(t)=C_{\text {ini }} \mathrm{e}^{-K t}
$$

where $K$ characterizes the overall degradation of species A in the whole system. The reaction rate constant in the reactor, $k$, is investigated from $K$ as

$$
k=\frac{K\left(t_{\mathrm{T}}+t_{\mathrm{R}}\right)-\ln \left(1-K t_{\mathrm{M}}\right)}{t_{\mathrm{R}}} .
$$

The assumptions as well as more details of the determination of the reaction rate constant can be found in Supporting Information File 1.

\section{Experimental methods Ultrasonic dispersion}

Two ultrasonic processors, Topas UDS751 (sonotrode S7) and Hielscher UP100H (sonotrode MS7), were employed to disperse the $1 \mathrm{~g} / \mathrm{L} \mathrm{TiO}_{2} \mathrm{P} 25$ suspensions [28]. The dispersed volumes were varied and the ultrasonic power was altered by varying the amplitude (20-100\%) and the immersion level of the sonotrodes $(2.0-5.5 \mathrm{~cm})$. The power was measured by a Voltcraf $^{\circledR}$ Energy Logger 4000. Samples were periodically taken for size characterization and transmittance measurement.

\section{Color removal of methylene blue}

The photocatalytic properties of $\mathrm{P} 25$ were examined by characterizing the discoloration of MB. The desired MB concentration $[6,7,29]$ of $0.01 \mathrm{mM}$ was obtained by adding the MB stock solution into the dispersed P25 suspensions. To achieve an adsorption-desorption equilibrium, the suspensions were stirred in dark for $30 \mathrm{~min}$. The discoloration was then performed in the illuminated flow reactor. Samples were taken at one-, five- or ten-minute intervals. The supernatant fluids were separated by an Eppendorf 5417 centrifuge and stored for further analysis. 
The process parameters of these experiments were adapted to different purposes: (1) verifying the kinetic model by altering the volume of the suspensions, (2) studying the influence of the P25 photocatalyst size by varying the degree of ultrasonic dispersion, and (3) investigating the effect of the turbidity of the suspensions by working with different P25 concentrations. All experiments were repeated two or three times to check the reproducibility.

\section{Analytical method}

The particle size distribution of the $\mathrm{TiO}_{2} \mathrm{P} 25$ suspensions was characterized by a Malvern Nano S90 photon correlation spectrometer [30]. The immediate results are the intensity-weighted distribution functions. Two parameters of analysis, the intensity-weighted harmonic mean size, $x_{\text {cum }}$, and the polydispersity index, PDI, were examined as a function of dispersion time.

The extinction coefficient of the P25 suspensions was calculated by means of the Beer-Lambert equation from the transmitted light measured with a Varian Cary 100 Bio UV-vis spectrometer [31]. The transmittance was investigated through $10 \mathrm{~mm}$ path length $\mathrm{P} 25$ suspensions with concentration in the range of $0.01-0.1 \mathrm{~g} / \mathrm{L}$ and with the aggregate size varying from 234-343 nm. Note that for the second suspension, the transmittance of the $100 \times$ diluted samples from the original $1 \mathrm{~g} / \mathrm{L}$ suspensions were measured.

In order to measure the amount of MB remaining in the solution for the discoloration tests, we measured the absorbance in the supernatant suspensions through $10 \mathrm{~mm}$ optical path length by UV-vis spectroscopy. The MB concentrations were calibrated from the absorbance at $\lambda=664 \mathrm{~nm}$ [19] (the calibration curve can be found in Figure S2 of Supporting Information File 1) and are plotted as a function of irradiation time.

\section{Results and Discussion Verification of the kinetic model}

In essence, an increase of the suspension amount in the mixing tank, which has no contribution to the reaction, results in a slower overall degradation rate constant, $K$, for the whole system, while the reaction rate constant, $k$, in the reactor remains unchanged as expected. Experimentally the downtrend of $K$ along with the variation of five suspension volumes (Figure 3) is attained.

Furthermore, the reaction rate constant, $k$, calculated from Equation 4 deviates within the upper and lower bands (Figure 3) and as predicted, yields an average value of $0.100 \pm$ $0.011 \mathrm{~min}^{-1}$. This result affirms the accuracy of the model, which eventually can support further studies with the new setup based on a PFR.

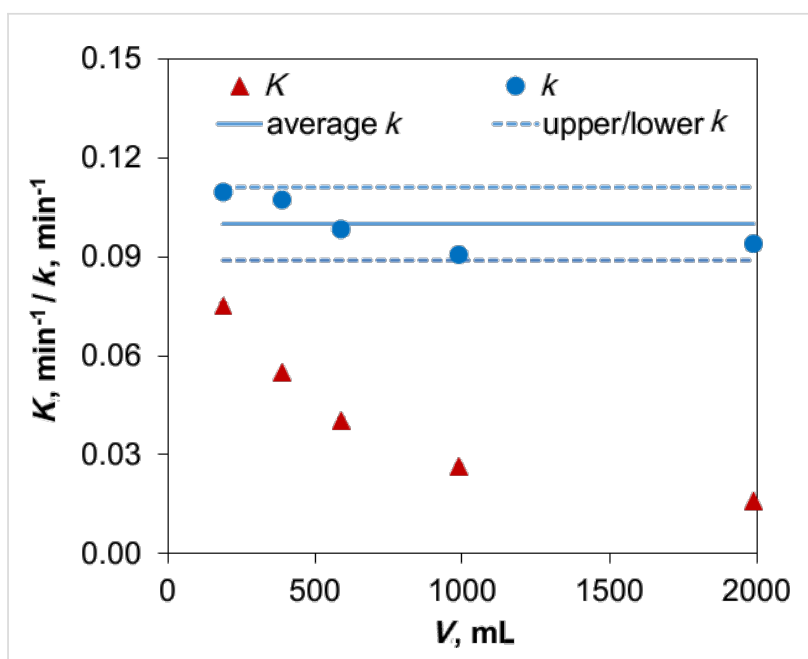

Figure 3: Apparent reaction rate constant $K$ for the whole system and the intrinsic reaction rate constant $k$ in the photoreactor, as a function of the volume of the mixing tank. Experiments were replicated twice and error bars of less than $2 \%$ were found, indicating the span between the minimum and maximum values are included in the markers and prove the precision of the data.

\section{Ultrasonic dispersion of $\mathrm{TiO}_{2}$ aggregates}

The photocatalyst P25 suspensions were dispersed with variable ultrasonic amplitudes and volumes. These different preparations allow the comparison of experiments from the aspect of electric consumption. Accordingly, the energy density, $E_{\mathrm{V}}$, defined as the integral of power consumption, $P$, by time, $t$, per volume unit, $V, E_{\mathrm{V}}=P \cdot t / V$ was considered.

The decrease of the intensity-weighted harmonic mean size over the energy density for independently prepared $\mathrm{TiO}_{2} \mathrm{P} 25$ suspensions is shown in Figure 4. Note that the power values refer to the real electric power consumption, which is not identical to the actual input of sound energy into the suspensions. Therefore, the dispersion results slightly deviate from each other.

Experimentally, an exponential decay in photocatalyst size is specified with a high coefficient of determination $\left(\mathrm{R}^{2}=\right.$ 0.96-0.99)

$$
x_{\mathrm{cum}} \propto E_{\mathrm{V}}^{-(0.13 \rightarrow 0.14)}
$$

and fits to an empirical energy density concept [32]

$$
x_{\text {cum }}=x_{\text {ref }}\left(\frac{E_{\mathrm{V}}}{E_{\mathrm{V}, \text { ref }}}\right)^{-\alpha},
$$

where $x_{\text {ref }}$ and $E_{\mathrm{V} \text {,ref }}$ denote the corresponding reference values, and the exponent $\alpha$ quantifies the efficiency of the dispersion 


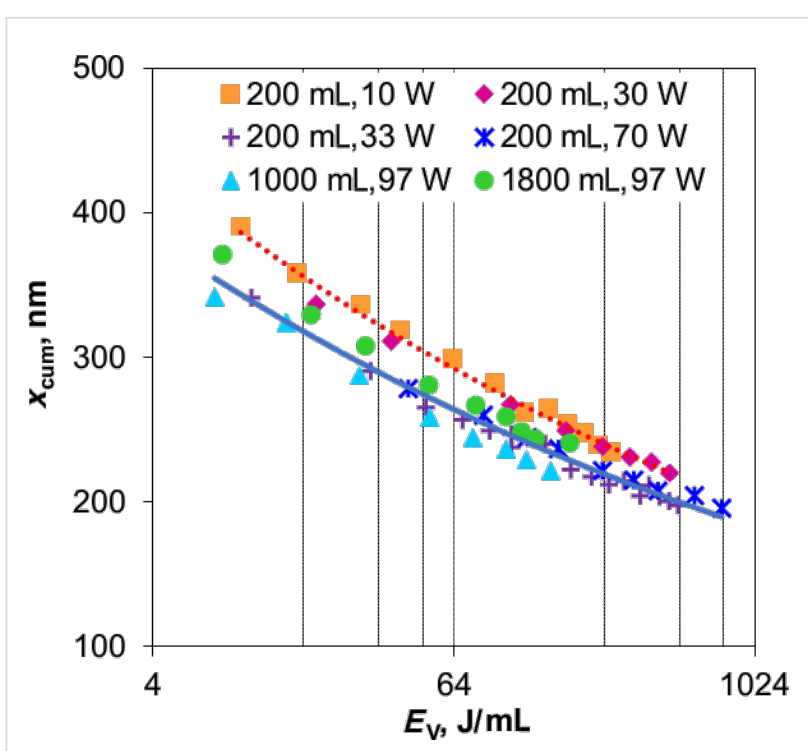

Figure 4: Aggregate size of $\mathrm{P} 25$ as a function of ultrasonic energy density performed by two ultrasonic devices, Hielscher UP100H (red dot) and Topas UDS751 (blue line).

procedure (i.e., small values mean that high energy densities are required for significant changes in the size distribution).

Equation 5 implies that to halve the $\mathrm{TiO}_{2} \mathrm{P} 25$ size requires an energy density change by a factor of 170 (corresponding to a time modification of a factor of 30 when generating a $100 \mathrm{~W}$ ultrasonication in a $1000 \mathrm{~mL}$ suspension). Consequently, this energy density concept can be used for further academic or economic estimation in the field of $\mathrm{TiO}_{2}$ dispersion. The influence of aggregate size on the interaction between photocatalyst and UV-vis illumination was simply tested. However, due to the very high turbidity of the original suspensions with a concentration of $1 \mathrm{~g} / \mathrm{L}$, the transmittances are extremely low for a differentiation (less than $0.16 \%$ in the UV range). The values of the $100 \times$ diluted samples are therefore substituted. As shown in Figure 5a, absorbance, scattering and other light phenomena in nano-colloidal suspensions [33] result in a loss of energy of the incident beam. In UV range, where radiation is adequate for $\mathrm{TiO}_{2}$ photocatalytic activation (appropriate for the photon energy of $3.2 \mathrm{eV}$ and a wavelength of $387 \mathrm{~nm}[2,22,34,35]$ ), the independence of transmittance as a function of the photocatalyst size is no longer valid as it is in the visible range. Transmittance tends to be restricted in finer suspensions as the result of a more effective absorbance [21]. A further evaluation also shows that at $\lambda=365 \mathrm{~nm}$, disaggregation of catalyst induces a $50 \%$ higher extinction coefficient, $\varepsilon$, corresponding to a $1.5 \times$ increase of active sites (Figure 5b). UV irradiation can eventually penetrate the smaller aggregates in suspensions without significant obscuration.

\section{Degradation experiments}

As discussed, fine aggregates in stable suspensions achieved by ultrasonic dispersion allow a more efficient photonic absorbance, and are hence expected to promote photocatalysis. Practically, a change of reaction rate constant of $1 \mathrm{~g} / \mathrm{L}$ P25 suspensions by $23 \%$ is achieved when reducing $x_{\text {cum }}$ from 380 to $250 \mathrm{~nm}$ (Figure 6), that is, the smaller particles/aggregates only have a slight influence on photocatalytic activity.

To consider the agglomerate size we approached the $90 \%$ quantile, $x_{90 \text {,int }}$, which is $90 \%$ of the intensity-weighted cumulative distribution. For a log-normal distribution $x_{90, \text { int }}$ is derived from the median of the intensity-weighted distribution function $x_{50, \text { int }}$ and the standard deviation, $\sigma_{\mathrm{LN}}[36,37]$ :

$$
\begin{gathered}
x_{90, \text { int }}=x_{50, \text { int }} \exp \left(1.282 \sigma_{\mathrm{LN}}\right) \\
x_{50, \text { int }}=x_{\text {cum }} \sqrt{1+\mathrm{PDI}} \\
\sigma_{\mathrm{LN}}=\sqrt{\ln (1+\mathrm{PDI})}
\end{gathered}
$$
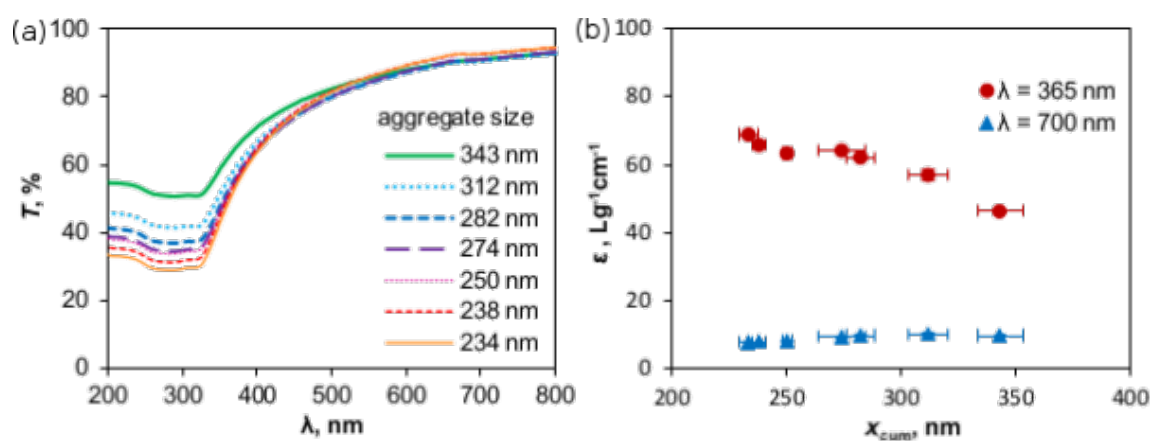

Figure 5: (a) Light transmittance $(T)$ through a $1 \mathrm{~cm}$ optical path length and (b) extinction coefficient $(\varepsilon)$ of $0.01 \mathrm{~g} / \mathrm{L} P 25$ suspensions with the variation of aggregate size (measurements were repeated three times and error bars indicate the standard deviation). 


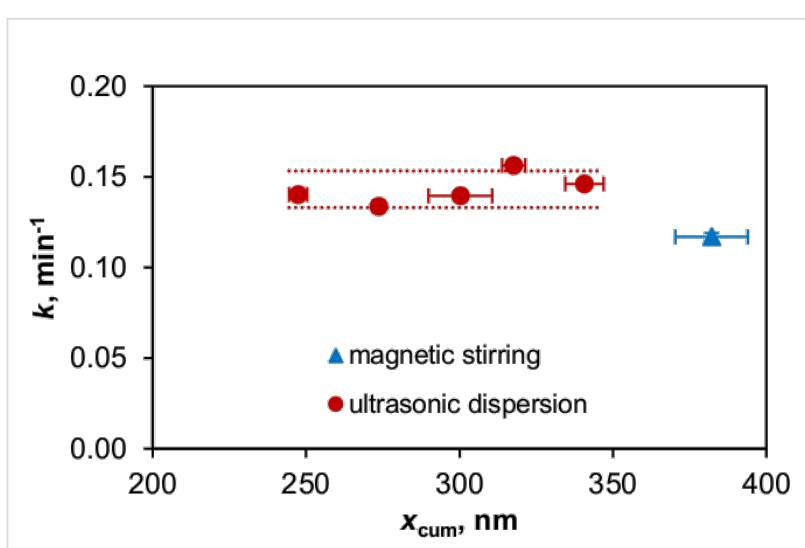

Figure 6: Dependence of MB discoloration in $1 \mathrm{~g} / \mathrm{L} \mathrm{P25}$ suspensions on the photocatalyst aggregate size achieved by ultrasonic dispersion and magnetic stirring. The experiments were repeated twice, and the error bars indicate the span between the minimum and maximum values, and the upper and lower bands indicate the t-confidence intervals of $95 \%$.

The influence of agglomerate size on MB degradation given in Supporting Information File 1 shows the same tendency as that of aggregate size. Interestingly, these two results prove that photocatalytic activity is not further increased beyond a certain degree of dispersion in spite of a more efficient interaction between irradiation and photocatalyst. Note that in this research, we used $1 \mathrm{~g} / \mathrm{L}$ P25 suspensions, which are considered to be the optimal concentration (Figure 7).

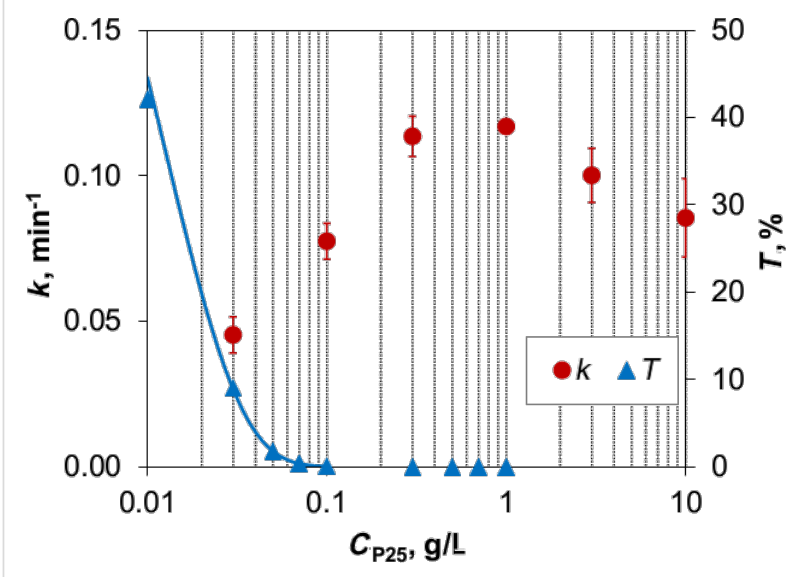

Figure 7: Influence of P25 catalyst concentration on the reaction rate constant and the transmittance through the thickness of the reactor at $\lambda=365 \mathrm{~nm}$ (experiments were repeated two or three times and error bars indicate the standard deviation).

In the range of low concentration, the higher photocatalyst loading produces an increase of the active sites resulting in a faster degradation. A further increase in the concentration limits the photocatalytic property. This can be explained by investigating the extinction coefficient of the P25 suspension (Figure 7). At $\lambda=365 \mathrm{~nm}, \varepsilon=36.65 \mathrm{Lg}^{-1} \mathrm{~cm}^{-1}$, which means that only $2.57 \%$ of this irradiation can penetrate a $1 \mathrm{~mm}$ path length of a $1 \mathrm{~g} / \mathrm{L}$ suspension or a $0.1 \mathrm{~mm}$ path length of a $10 \mathrm{~g} / \mathrm{L}$ suspension. Since the thickness of the reactor is $22 \mathrm{~mm}$, very little irradiation travels through such exceedingly turbid suspensions. For this reason, the disintegration of the catalyst in the $1 \mathrm{~g} / \mathrm{L}$ suspension in spite of varying the size causes the overturbidity $[5,10]$, resulting in the insignificant enhancement of MB discoloration.

\section{Conclusion}

This study addressed the photocatalysis performance of suspended catalysts in an aggregated state. In particular, we examined to what degree the state of dispersion of aggregated $\mathrm{TiO}_{2}$ nanoparticles (P25) affects the photodegradation of methylene blue.

For this purpose, a lab-scale plug flow reactor was designed, which facilitates a defined variation of process parameters such as intensity of UV light, optical path length and flow regime. The apparent reaction rate constant of such a reactor can be easily translated into an intrinsic reaction rate constant when the material balance between the storage tank and the reactor is established. The proposed calculation scheme was experimentally verified.

In addition, the study took a closer look at the dispersion procedures for the $\mathrm{TiO}_{2}$ suspensions. Focus was placed on ultrasonication, which yields highly intense hydrodynamic and thermal stresses and thus allows for a significant disintegration of particle aggregates. We showed that the energy density concept works well for comparing the dispersion performance of different ultrasonic processors and facilitates the comparison of dispersion procedures between different laboratories.

The central part of the paper was the study of the discoloration of MB in the presence of P25 under UV illumination. The size of the aggregates appeared to have only a minor influence on the intrinsic reaction rate constant, even though the efficiency of photon absorbance increases with further dispersion. Obviously, the maximum reaction rate is already achieved after short ultrasonication time, which disperses the large micrometer-sized agglomerates into submicron aggregates. This outcome is explained by the limited UV penetration depth into the concentrated catalyst suspensions. In this regard it may not be surprising, yet from a practical point of view, it may help to reduce the energy consumption in the preparation of photocatalyst suspensions and to optimize their total particle concentration. 


\section{Supporting Information}

Details of a model for determination of the reaction rate constant in the experimental setup based on a PFR, the calibration of $\mathrm{MB}$, the stability test of suspensions, the results of photon correlation spectroscopy, and the impact of agglomerate size on the discoloration of $\mathrm{MB}$ in photocatalysis can be found in this file.

\section{Supporting Information File 1}

Details for the reaction rate model and experimental setup [http://www.beilstein-journals.org/bjnano/content/ supplementary/2190-4286-6-250-S1.pdf]

\section{Acknowledgement}

This work is funded by the Erasmus Mundus - One More Step program, the Federal Ministry of Education and Research (BMBF) and the CLIENT/Navitex project 02WCL1264A. We thank Felix Alscher for the experimental work, Jochen Förster for the technical help and acknowledge fruitful discussions with Hagen Eckert, Sara Teixeira and Robert Langsch.

\section{References}

1. Mohajerani, M.; Mehrvar, M.; Ein-Mozaffari, F. Int. J. Eng. 2009, 3, 120-146.

2. Ochiai, T.; Fujishima, A. J. Photochem. Photobiol., C: Photochem. Rev. 2012, 13, 247-262. doi:10.1016/j.jphotochemrev.2012.07.001

3. Forgacs, E.; Cserháti, T.; Oros, G. Environ. Int. 2004, 30, 953-971. doi:10.1016/j.envint.2004.02.001

4. Rajeshwar, K.; Osugi, M. E.; Chanmanee, W.; Chenthamarakshan, C. R.; Zanoni, M. V. B.; Kajitvichyanukul, P.; Krishnan-Ayer, R. J. Photochem. Photobiol., C: Photochem. Rev. 2008, 9, 171-192. doi:10.1016/j.jphotochemrev.2008.09.001

5. Bodzek, M.; Rajca, M. Ecol. Chem. Eng. S 2012, 19, 489-512. doi:10.2478/v10216-011-0036-5

6. Xu, N.; Shi, Z.; Fan, Y.; Dong, J.; Shi, J.; Hu, M. Z.-C. Ind. Eng. Chem. Res. 1999, 38, 373-379. doi:10.1021/ie980378u

7. Houas, A.; Lachheb, H.; Ksibi, M.; Elaloui, E.; Guillard, C.; Herrmann, J.-M. Appl. Catal., B: Environ. 2001, 31, 145-157. doi:10.1016/S0926-3373(00)00276-9

8. Knowles, J. P.; Elliott, L. D.; Booker-Milburn, K. I. Beilstein J. Org. Chem. 2012, 8, 2025-2052. doi:10.3762/bjoc.8.229

9. Herrmann, J.-M. Appl. Catal., B: Environ. 2010, 99, 461-468. doi:10.1016/j.apcatb.2010.05.012

10. Thakur, R. S.; Chaudhary, R.; Singh, C. J. Renewable Sustainable Energy 2010, 2, 042701. doi:10.1063/1.3467511

11. Ohtani, B. Design and Development of Active Titania and Related Photocatalysts. In Photocatalysis and Water Purification: From Fundamentals to Recent Applications; Pichat, P., Ed.; Wiley-VCH: Weinheim, Germany, 2013; pp 75-102. doi:10.1002/9783527645404.ch4
12. Harra, J.; Nikkanen, J.-P.; Aromaa, M.; Suhonen, H.; Honkanen, M.; Salminen, T.; Heinonen, S.; Levänen, E.; Mäkelä, J. M. Powder Technol. 2013, 243, 46-52. doi:10.1016/j.powtec.2013.03.027

13. Nickel, C.; Angelstorf, J.; Bienert, R.; Burkart, C.; Gabsch, S.; Giebner, S.; Haase, A.; Hellack, B.; Hollert, H.; Hund-Rinke, K.; Jungmann, D.; Kaminski, H.; Luch, A.; Maes, H. M.; Nogowski, A.; Oetken, M.; Schaeffer, A.; Schiwy, A.; Schlich, K.; Stintz, M.; von der Kammer, F.; Kuhlbusch, T. A. J. J. Nanopart. Res. 2014, 16, 1-12. doi:10.1007/s11051-014-2260-2

14. Cabrera, M. I.; Alfano, O. M.; Cassano, A. E. J. Phys. Chem. 1996, 100, 20043-20050. doi:10.1021/jp962095q

15. Jassby, D.; Budarz, J. F.; Wiesner, M. Environ. Sci. Technol. 2012, 46, 6934-6941. doi:10.1021/es202009h

16. Amano, F.; Ishinaga, E.; Yamakata, A. J. Phys. Chem. C 2013, 117, 22584-22590. doi:10.1021/jp408446u

17. Kočí, K.; Obalová, L.; Matějová, L.; Plachá, D.; Lacný, Z.; Jirkovský, J.; Šolcová, O. Appl. Catal., B: Environ. 2009, 89, 494-502. doi:10.1016/j.apcatb.2009.01.010

18. Moradi Golsheikh, A.; Lim, H. N.; Zakaria, R.; Huang, N. M. RSC Adv. 2015, 5, 12726-12735. doi:10.1039/C4RA14775H

19. Swedish Standards Institute; Photocatalytic activity of surfaces Determination of photocatalytic activity by degradation of methylene blue; DIN 52980, Oct 2008.

http://www.sis.se/en/manufacturing-engineering/surface-treatment-andcoating/surface-treatment/din-52980

20. Braham, R. J.; Harris, A. T. Ind. Eng. Chem. Res. 2009, 48, 8890-8905. doi:10.1021/ie900859z

21. Braslavsky, S. E.; Braun, A. M.; Cassano, A. E.; Emeline, A. V.; Litter, M. I.; Palmisano, L.; Parmon, V. N.; Serpone, N. Pure Appl. Chem. 2011, 83, 931-1014. doi:10.1351/PAC-REC-09-09-36

22. Chong, M. N.; Jin, B.; Chow, C. W. K.; Saint, C. Water Res. 2010, 44, 2997-3027. doi:10.1016/j.watres.2010.02.039

23. Bayarri, B.; Giménez, J.; Maldonado, M. I.; Malato, S.; Esplugas, S. Chem. Eng. J. 2013, 232, 405-417. doi:10.1016/j.cej.2013.07.102

24. Malato, S.; Blanco, J.; Alarcón, D. C.; Maldonado, M. I.; Fernández-lbáñez, P.; Gernjak, W. Catal. Today 2007, 122, 137-149. doi:10.1016/j.cattod.2007.01.034

25. McCullagh, C.; Skillen, N.; Adams, M.; Robertson, P. K. J. J. Chem. Technol. Biotechnol. 2011, 86, 1002-1017. doi:10.1002/jctb.2650

26. Tanveer, M.; Tezcanli Guyer, G. Renewable Sustainable Energy Rev. 2013, 24, 534-543. doi:10.1016/j.rser.2013.03.053

27. Davis, M. E.; Davis, R. J. The Basics of Reaction Kinetics for Chemical Reaction Engineering. In Fundamentals of Chemical Reaction Engineering; Glandt, E. D.; Klein, M. T.; Edgar, T. F., Eds.; McGraw-Hill Higher Education: New York, 2003; pp 1-52.

28. Suslick, K. S.; Price, G. J. Annu. Rev. Mater. Sci. 1999, 29, 295-326. doi:10.1146/annurev.matsci.29.1.295

29. Eckert, H.; Bobeth, M.; Teixeira, S.; Kühn, K.; Cuniberti, G. Chem. Eng. J. 2015, 261, 67-75. doi:10.1016/j.cej.2014.05.147

30. ISO; Particle Size Analysis - Photon Correlation Spectroscopy; DIN ISO 13321, Oct 2004. http://www.iso.org/iso/iso_catalogue/catalogue_tc/catalogue_detail.htm ?csnumber $=21707$

31. Gregory, J. Colloid Interface Sci. 2009, 147-148, 109-123. doi:10.1016/j.cis.2008.09.003

32. Pohl, M.; Schurchmann, H. P.; Schubert, H. Chem. Ing. Tech. 2005, 77, 258-262. doi:10.1002/cite.200407020 
33. Dörfler, H.-D. Lichtstreuung an kolloiden und makromolekularen Systemen. Grenzflächen- und Kolloidchemie; Wiley-VCH: Weinheim, Germany, 1994; pp 412-451.

34. Ishibashi, K.; Fujishima, A.; Watanabe, T.; Hashimoto, K. J. Photochem. Photobiol., A: Chem. 2000, 134, 139-142. doi:10.1016/S1010-6030(00)00264-1

35. Linsebigler, A. L.; Lu, G.; Yates, J. T. Chem. Rev. 1995, 95, 735-758. doi:10.1021/cr00035a013

36. Babick, F.; Vorbau, M.; Stintz, M. Part. Part. Syst. Charact. 2012, 29 , 116-127. doi:10.1002/ppsc.201000025

37. Rumpf, $H$. The characteristics of systems and their changes of state. In Particle Technology; Scarlett, B., Ed.; Chapman and Hall, 2013; pp 8-55.

\section{License and Terms}

This is an Open Access article under the terms of the Creative Commons Attribution License

(http://creativecommons.org/licenses/by/2.0), which permits unrestricted use, distribution, and reproduction in any medium, provided the original work is properly cited.

The license is subject to the Beilstein Journal of

Nanotechnology terms and conditions:

(http://www.beilstein-journals.org/bjnano)

The definitive version of this article is the electronic one which can be found at: doi:10.3762/bjnano.6.250 